\title{
Chaos synchronization in long-range coupled map lattices
}

\author{
C. Anteneodo ${ }^{1}$, A.M. Batista ${ }^{2}$ and R.L. Viana ${ }^{3}$ \\ 1. Centro Brasileiro de Pesquisas Fúsicas, \\ Rua Dr. Xavier Sigaud 150, 22290-180, Rio de Janeiro, RJ, Brazil; \\ 2. Departamento de Matemática e Estatística, Universidade Estadual de Ponta Grossa, \\ 84030-900, Ponta Grossa, PR, Brazil; \\ 3. Departamento de Física, Universidade Federal do Paraná, \\ 81531-990, Curitiba, PR, Brazil.
}

\begin{abstract}
We investigate the synchronization phenomenon in coupled chaotic map lattices where the couplings decay with distance following a power-law. Depending on the lattice size, the coupling strength and the range of the interactions, complete chaos synchronization may be attained. The synchronization domain in parameter space can be analytically delimited by means of the condition of negativity of the largest transversal Lyapunov exponent. Here we analyze in detail the role of all the system parameters in the ability of the lattice to achieve complete synchronization, testing analytical results with the outcomes of numerical experiments.
\end{abstract}

PACS numbers: 05.45.Ra,05.45.-a,05.45.Xt

Coupled map lattices (CMLs), dynamical systems with discrete space and time, are being intensively investigated nowadays as models of spatiotemporal phenomena occurring in a wide variety of systems of physical, biological and technical interest [1]. In this letter we will deal with the phenomenon of synchronization and, in particular, amongst the various kinds of synchronized behavior, with the complete synchronization (CS) [2] occurring in CMLs with regular long-range interactions. Most work done so far on synchronization in CMLs has focused on two extreme coupling types: local (nearest-neighbor) [3] and global ("mean field") ones [4]. However, non-local couplings are relevant to a variety of situations ranging from neural networks [5] to physico-chemical reaction systems [6].

We consider chains of $N$ coupled one-dimensional (1D) chaotic maps $x \mapsto f(x)$ whose evolution is given by [7]

$x_{n+1}^{(i)}=(1-\varepsilon) f\left(x_{n}^{(i)}\right)+\frac{\varepsilon}{\eta} \sum_{r=1}^{N^{\prime}} \frac{f\left(x_{n}^{(i-r)}\right)+f\left(x_{n}^{(i+r)}\right)}{r^{\alpha}}$,

where $x_{n}^{(i)}$ represents the state variable for the site $i$ $(i=1,2, \ldots, N)$ at time $n, \varepsilon \geq 0$ is the coupling strength, $\alpha \geq 0$ controls the effective range of the interactions and $\eta=2 \sum_{r=1}^{N^{\prime}} r^{-\alpha}$ is a normalization factor, with $N^{\prime}=(N-1) / 2$ for odd $N$. The main interest in this coupling scheme resides in the fact that it allows to investigate the role of the range of the interactions, scanning from the local $(\alpha \rightarrow \infty)$ to the global $(\alpha=0)$ cases [8].

CS takes place when the dynamical variables that define the state of each map adopt the same value for all the coupled maps at all times $n$, i.e, $x_{n}^{(1)}=x_{n}^{(2)}=\ldots=$ $x_{n}^{(N)} \equiv x_{n}^{(*)}$. Depending on the lattice size and on the range of the interactions, there may exist an interval of values of the coupling strength $\varepsilon$, for which such state is spontaneously attained, as we have analytically shown in previous work [9]. It is our purpose here to scrutinize the role of all the system parameters in the ability of the lattice to synchronize. Analytical results will be compared with the outputs of numerical simulations performed for diverse chaotic maps.

Complete synchronization can be characterized by a complex order parameter defined, for time $n$, as $R_{n}=$ $\left|\frac{1}{N} \sum_{j=1}^{N} e^{2 \pi i x_{n}^{(j)}}\right|[10]$. Typically a time-averaged amplitude $\bar{R}$ is computed over a time interval long enough to allow the lattice attain the asymptotic state. In the CS state, one has $\bar{R}=1$ within a small allowed deviation.

Another diagnostic of complete synchronization can be obtained from the Lyapunov spectrum (LS) of the CS states. If the chaotic maps are completely synchronized, the maximal Lyapunov exponent, in the direction parallel to the synchronization manifold (SM), is strictly positive. The negativity of the second largest Lyapunov exponent, which belongs to the direction transversal to the SM, indicates the stability of the synchronized state under small transversal displacements [11].

In our case the lattice dynamics given by Eq. (1) can be written as $x_{n+1}^{(i)}=\sum_{j} F_{i j} f\left(x_{n}^{(j)}\right)$, where $\mathbf{F}$ is a matrix of the form

$$
\mathbf{F}=\left[(1-\varepsilon) \mathbb{1}+\frac{\varepsilon}{\eta} \mathbf{B}\right],
$$

with $\mathbb{1}$ the $N \times N$ identity matrix and $\mathbf{B}$ defined by $B_{j k}=\left(1-\delta_{j k}\right) / r_{j k}^{\alpha}$, being $r_{j k}=\min _{l \in \mathcal{Z}}|j-k+l N|$.

The Lyapunov spectrum is obtained from the dynamics of tangent vectors $\xi$, which in turn is obtained by differentiation of the original evolution equations. In matrix form the tangent dynamics reads $\xi_{n}=\mathcal{T}_{n} \xi_{0}$, where $\mathcal{T}_{n}$ is product of $n$ Jacobian matrices calculated at successive points of a given trajectory. If $\Lambda^{(1)}, \ldots, \Lambda^{(N)}$ are the eigenvalues of $\hat{\Lambda}=\lim _{n \rightarrow \infty}\left(\mathcal{T}_{n}^{T} \mathcal{T}_{n}\right)^{\frac{1}{2 n}}$, the Lyapunov exponents are obtained as $\lambda^{(k)}=\ln \Lambda^{(k)}$, for $k=1, \ldots, N$ [12]. For the CS states, one arrives at the following expression for the Lyapunov spectrum [9] 


$$
\bar{\lambda}^{(k)}=\lambda_{U}+\ln \left|1-\varepsilon+\varepsilon \frac{b^{(k)}}{\eta}\right|,
$$

where $\lambda_{U}>0$ is the Lyapunov exponent of the uncoupled chaotic map, and $b^{(k)}$ are the eigenvalues of $\mathbf{B}$ that can be obtained by Fourier diagonalization and, for odd $N$, read

$$
b^{(k)}=2 \sum_{m=1}^{N^{\prime}} \frac{\cos (2 \pi k m / N)}{m^{\alpha}}, \quad 1 \leq k \leq N .
$$

For even $N$, summations run up to $N^{\prime}=N / 2$ and half of the $N^{\prime}$ th term has to be substracted. The maximal eigenvalue is $b^{(N)}=\eta$ and the minimal one is $b^{\left(N^{\prime}\right)}$. Except for the cases $k=N^{\prime}$, with even $N$, and $k=N$, the remaining eigenvalues are two-fold degenerate, being $b^{(k)}=b^{(N-k)}$.

In the calculation of Lyapunov exponents $\lambda^{(k)}$, notice that the parameters that define the particular uncoupled map affect only $\lambda_{U}$, while the second term in Eq. (3) is determined by the particular cyclic dependence on distance in the regular coupling scheme (a power law in our case). It can be easily verified that, for arbitrary $\alpha$, the CS state lies along the direction of the eigenvector associated to the largest exponent $\bar{\lambda}^{(N)}$. Therefore, the CS state will be transversally stable if the $(N-1)$ remaining exponents are negative, that is $\left|1-\varepsilon+\varepsilon b^{(k)} / \eta\right|<\mathrm{e}^{-\lambda_{U}}$, $\forall k \neq N$. This is equivalent to requiring that the second largest (or largest transversal) asymptotic exponent, denoted by $\bar{\lambda}^{\perp}$, be negative. This exponent is obtained from Eq. (3) with either $k=1$ (hence also $k=N-1$ due to degeneracy) or with $k=N^{\prime}$ (hence also $k=N^{\prime}+1$ if $N$ is odd), depending on whether $\left|1-\varepsilon+\varepsilon b^{(1)} / \eta\right|$ is, respectively, greater or smaller than $\left|1-\varepsilon+\varepsilon b^{\left(N^{\prime}\right)} / \eta\right|$. The condition $\bar{\lambda}^{\perp}<0$ leads to $\varepsilon_{c}<\varepsilon<\varepsilon_{c}^{\prime}$ [9] (see also [13]), where

$$
\begin{aligned}
& \varepsilon_{c}\left(\alpha, N, \lambda_{U}\right)=\left(1-\mathrm{e}^{-\lambda_{U}}\right)\left(1-\frac{b^{(1)}}{\eta}\right)^{-1} \text { and } \\
& \varepsilon_{c}^{\prime}\left(\alpha, N, \lambda_{U}\right)=\left(1+\mathrm{e}^{-\lambda_{U}}\right)\left(1-\frac{b^{\left(N^{\prime}\right)}}{\eta}\right)^{-1} .
\end{aligned}
$$

In Fig. 1 we show a variety of critical curves in parameter space $(\alpha, \varepsilon)$ obtained for different lattice sizes. Stable CS states dwell in the region bounded by the $\alpha=0$ axis, and two curve segments. The critical curves were obtained analytically from Eqs. (5) (lower curve) and (6) (upper curve). The symbols shown stand for the numerical results determined from the condition $\bar{R}=1$ with a tolerance of $10^{-6}$. Two different values of $\lambda_{U}$ were considered. Numerical results shown in Fig. 1 were computed for the piecewise linear (a) Bernoulli shift $f(x)=2 x$ $(\bmod 1)$ (therefore $\left.\lambda_{U}=\ln 2\right)$ and $(b)$ triangular map [14]

$$
f_{w}(x)=\left\{\begin{array}{cc}
x / w & \text { for } 0 \leq x \leq w \\
(1-x) /(1-w) & \text { for } w<x \leq 1
\end{array}\right.
$$
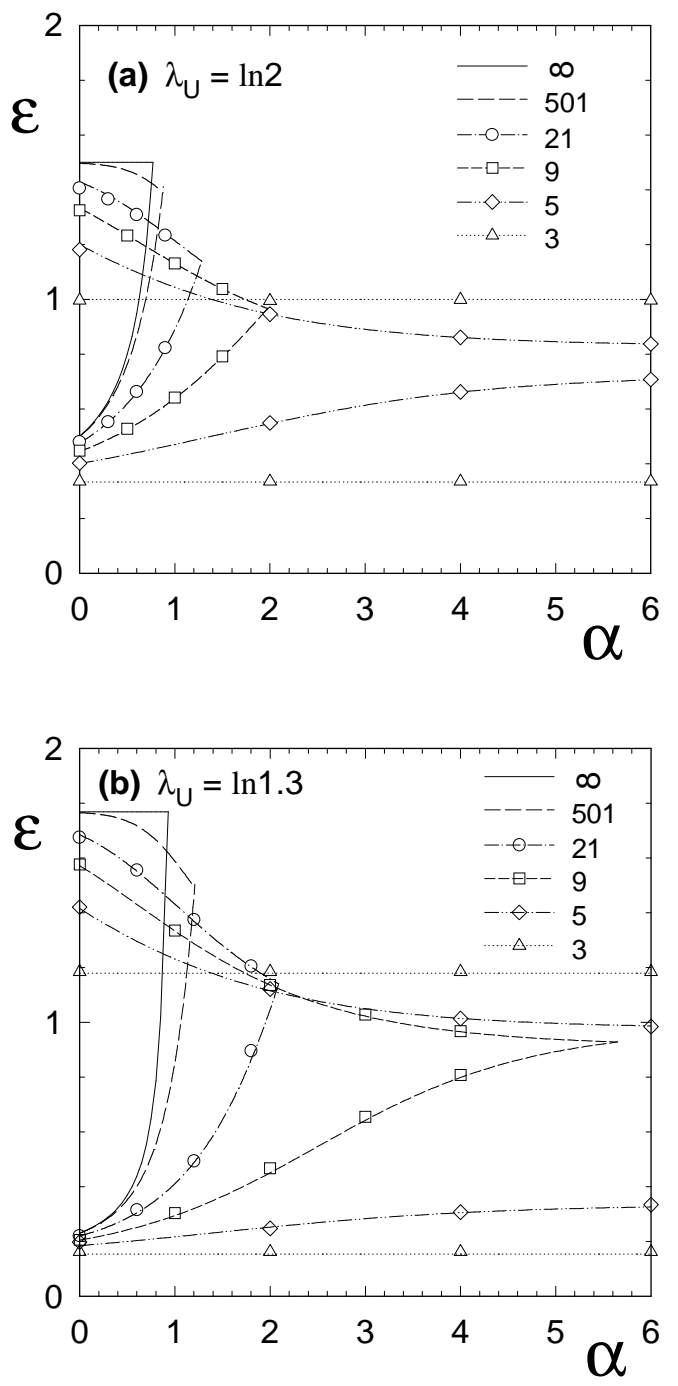

FIG. 1. Synchronization diagram in parameter space $(\alpha, \varepsilon)$, for different values of $N$ and $\lambda_{U}=\ln 2$ (a), $\ln 1.3$ (b). Lines correspond to analytical predictions; symbols to numerical simulations using the Bernoulli (a) or triangular (b) maps. Synchronization is transversally stable in the region between the couple of curves for each set of values of the parameters.

that for $w=0.074$ yields $\lambda_{U} \simeq \ln 1.3$ (notice that $\left.\lambda_{U}=-w \ln w-(1-w) \ln [1-w]\right)$. Additional tests (results not shown here) were performed for other maps such as the logistic map $f(x)=\mu x(1-x)$ with $\mu=4$ (hence $\lambda_{U}=\ln 2$ ) and $\mu=3.6533$ (hence $\lambda_{U} \simeq \ln 1.30$ ) yielding the same degree of agreement. For these interval maps, in principle, one must have $0 \leq \varepsilon \leq 1$ in order to guarantee that the state variables $x_{n}^{(j)}$ will remain inside the interval $[0,1]$. But, reinjection into the interval can be performed through an operation, for instance (mod 1), such that it does not spoil the Lyapunov exponent of the chaotic uncoupled map. If trajectory points were 
not reinjected, one can still look at our results as valid for trajectories or trajectory segments as long as the state variables remain confined within the given interval. Anyway, for other maps such as $f(x)=\exp \left\{-[(x-0.5) / \sigma]^{2}\right\}$ [15], one may have any coupling $\varepsilon \geq 0$ since the map is naturally defined in the full real axis. Tests performed with this Gaussian map (results not shown in this letter) are also in good accord with theoretical predictions.

In general terms, we observe that for weak coupling, the maps do not synchronize. As the coupling strength increases, synchronization can occur depending on the lattice parameters $\left(\alpha, N, \lambda_{U}\right)$. However, a too high coupling intensity $\varepsilon>\varepsilon_{c}^{\prime}$ has a destabilizing influence on the CS state and the lattice no longer synchronizes.

Concerning lattice size, already Fig. 1 exhibits the intuitive fact that it is more difficult to synchronize a larger lattice than a shorter one, all other parameters being kept fixed. In the limit $N \rightarrow \infty$ we obtain

$$
\varepsilon_{c}\left(\alpha, \infty, \lambda_{U}\right)=\frac{1-\mathrm{e}^{-\lambda_{U}}}{1-C(\alpha)}
$$

where $C(\alpha)=\lim _{N \rightarrow \infty} b^{(1)} / \eta$. This limit is equal to one for $\alpha>1$, so that Eq. (8) yields a divergent result. For $\alpha$ outside the domain of convergence of the series, i.e. $\alpha<1$, one gets

$$
C(\alpha)=\frac{1-\alpha}{\pi^{1-\alpha}} \int_{0}^{\pi} \frac{\cos (x)}{x^{\alpha}} d x .
$$

In that same range of $\alpha$ one has

$$
\varepsilon_{c}^{\prime}\left(\alpha, \infty, \lambda_{U}\right)=1+\mathrm{e}^{-\lambda_{U}}
$$

which is independent on $\alpha$, thus it yields a straight line in the plots of Fig. 1. From the intersection of $\varepsilon_{c}\left(\alpha, \infty, \lambda_{U}\right)$ with $\varepsilon_{c}^{\prime}\left(\alpha, \infty, \lambda_{U}\right)$ it results a critical value of the interaction range $\alpha_{c}$, such that, for $\alpha \leq \alpha_{c}<1\left(\alpha_{c}<d\right.$ in the $d$-dimensional case [9]), synchronization is possible even in the thermodynamic limit $N \rightarrow \infty$ for an appropriate window of $\varepsilon$. Observe the corresponding domains in Fig. 1.

As $N$ diminishes, the upper curve in Fig. 1, which is a straight line for infinite $N$, gains a negative inclination and extends for large $\alpha$ to values of $\varepsilon$ less than unity, indicating that the destabilizing effect of very strong coupling is more easily attained. The lower curve segment has a positive inclination, connecting to the upper curve at a point that forms a cusp for small $N$. For still smaller $N$ (e.g., $N \leq 5$ for $\lambda_{U}=\ln 2$ and $N \leq 8$ for $\lambda_{U}=\ln 1.3$ ) the two critical curves do not intersect each other even in the limiting case of first neighbors $(\alpha \rightarrow \infty)$.

The effect of lattice size can also be observed in Fig. 2 that exhibits the synchronization domains in the plane $(N, \varepsilon)$ for different values of $\alpha$. Similar plots have been observed in scale-free networks [16], as if there were an average or effective $\alpha$ in such cases. While for $\alpha>\alpha_{c}$ there is un upper bound $N_{b}\left(\alpha, \lambda_{U}\right)$ of the number of maps for which synchronization occurs; for $\alpha<\alpha_{c}$ any number of maps synchronize (because the critical curves in Fig. 2 do not intersect). Generically it is easier to synchronize a small number of maps. Consistently with this observation, lattices of small size (e.g., $N \leq 5$ for $\lambda_{U}=\ln 2$ ) can synchronize for any $\alpha$, for a certain window of $\varepsilon$ that narrows with increasing $\alpha$. For $N \leq 3$ there is, naturally, no dependence on $\alpha$ and the lattice syncronizes for any $\lambda_{U}>0$.

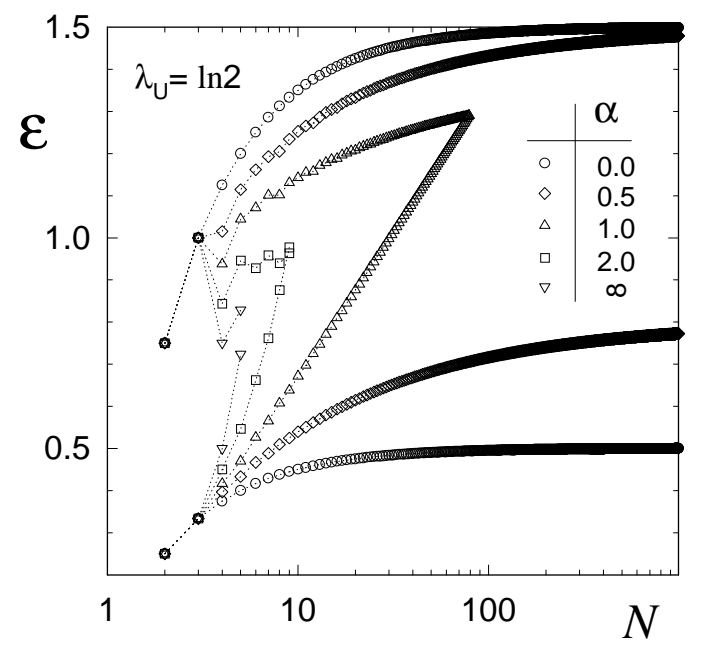

FIG. 2. Synchronization critical lines in the plane $(N, \varepsilon)$ for different values of $\alpha$ and $\lambda_{U}=\ln 2$. In this case $\alpha_{c} \approx 0.77$. Symbols correspond to theoretical calculations. Dotted lines are guides to the eyes. Synchronization is transversally stable in the region between the two curves for each set of values of the parameters.

Although Figs. 1(a) and 1(b) yield qualitative similar results, their comparison makes clear that, as expected, the more chaotic the uncoupled maps are, the more difficult becomes to obtain their synchronization. The influence of the Lyapunov exponent $\lambda_{U}$ on the synchronization domains in the parameter space $(\alpha, \varepsilon)$ is displayed in Fig. 3. The exhibited numerical results were acquired for $N=21$ either Bernoulli or triangular maps.

If the positive Lyapunov exponent of the uncoupled map increases, the synchronization domain shrinks, collapsing in the limit $\lambda_{U} \rightarrow \infty$. In the opposite limit of $\lambda_{U} \rightarrow 0^{+}$one gets

$$
\begin{array}{ll}
\varepsilon_{c} \rightarrow 0 & \text { and } \\
\varepsilon_{c}^{\prime} \rightarrow 2\left(1-\frac{b^{\left(N^{\prime}\right)}}{\eta}\right)^{-1} .
\end{array}
$$

This limit value of $\varepsilon_{c}^{\prime}$ depends on $\alpha$ and $N$. If $N \rightarrow \infty$ and $\alpha \rightarrow 0(\infty), \varepsilon_{c}^{\prime}$ goes to 2.0 (1.0) in the limit of vanishing chaos. (Although $N=21$, all these extreme behaviors are already insinuated in Fig. 3.) As a consequence, the critical value $\alpha_{c}<1$ below which the lattice synchronizes in the thermodynamic limit depends on the degree 
of chaoticity of the uncoupled maps. This dependence can be explicitly obtained by inversion of

$$
\lambda_{U}=\ln \left[\frac{2}{C\left(\alpha_{c}\right)}-1\right]
$$

extracted from Eqs. (8) and (10). The critical value $\alpha_{c}$ as a function of $\lambda_{U}$ is displayed in Fig. 4 . In the limit of vanishing (infinite) $\lambda_{U}, \alpha_{c}$ goes $1.0(0.0)$.

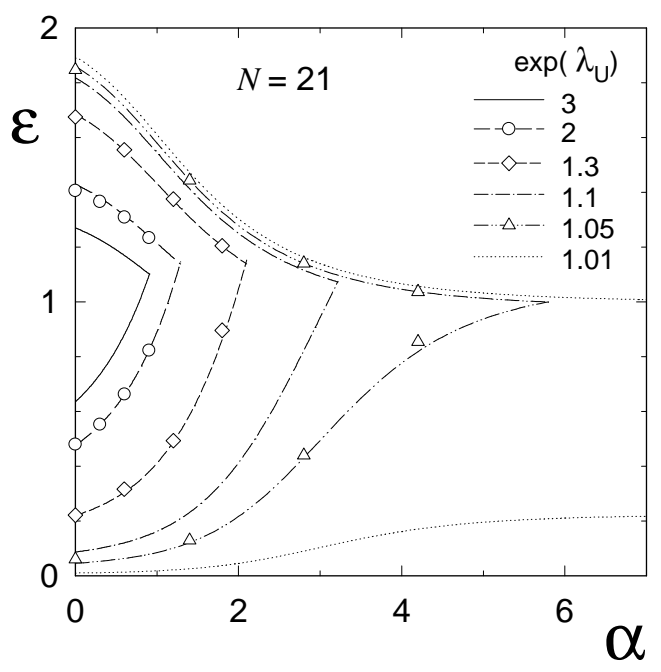

FIG. 3. Synchronization domains in parameter plane $(\alpha, \varepsilon)$ for 21 coupled chaotic maps and various values of $\lambda_{U}$. Numerical simulations were performed for Bernoulli or triangular maps. Critical curves were obtained analytically from Eqs. (5) and (6).

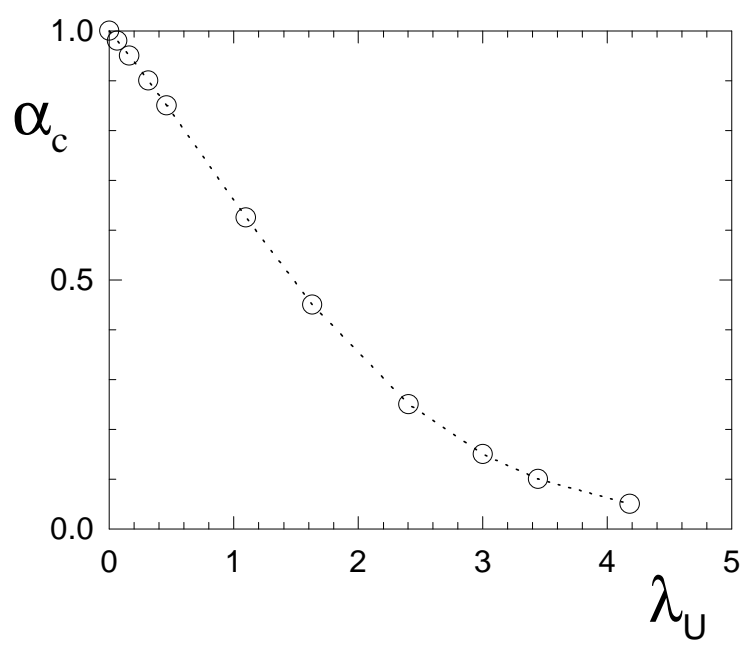

FIG. 4. Critical value $\alpha_{c}$, below which synchronization is stable even in the thermodynamic limit, as a function of $\lambda_{U}$ (symbols), determined from Eq. (13). The dotted line is a guide to the eyes.
When $\alpha>\alpha_{c}$, it must be $N \leq N_{b}\left(\alpha, \lambda_{U}\right)$ for the lattice to synchronize, where $N_{b}$ decreases with increasing $\alpha-\alpha_{c}$ (as shown in Fig 2). In the limit $\alpha \rightarrow \infty$, it is easy to obtain, from the condition $\varepsilon_{c}^{\prime}>\varepsilon_{c}$, an approximate expression for the maximal size, valid when one has sufficiently small $\lambda_{U}$ and large $N_{b}$ :

$$
N_{\max } \equiv N_{b}\left(\infty, \lambda_{U}\right) \leq \pi \sqrt{\frac{2}{\lambda_{U}}} .
$$

In Fig. 5, we exhibit the maximal size $N_{\max }$ for which synchronization can be achieved in the limit of nearestneighbor couplings (hence for any $\alpha$ ) as a function of $\lambda_{U}$, together with the approximation given by Eq. (14).

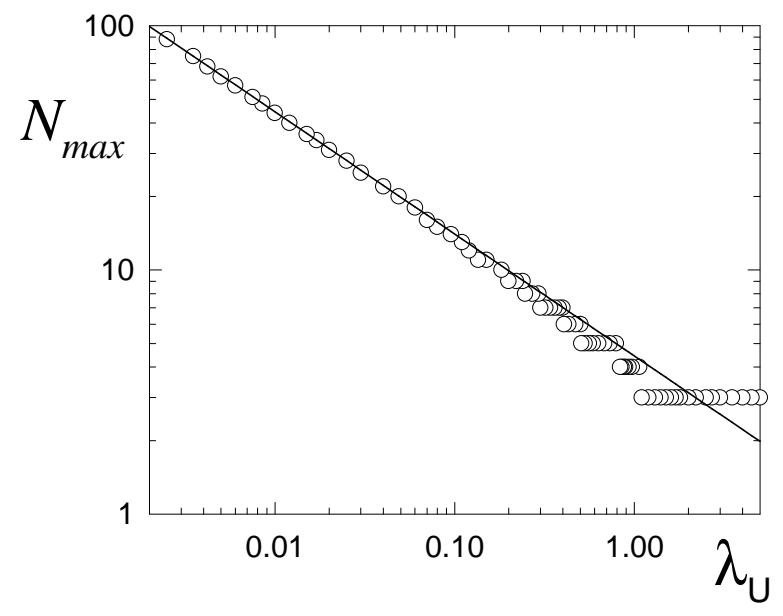

FIG. 5. Maximal lattice size $N_{\max } \equiv N_{b}\left(\alpha, \lambda_{U}\right)$, for which synchronization can be achieved for any $\alpha$, as a function of the chaoticity indicator $\lambda_{U}$ (circles), determined from the condition $\varepsilon_{c}^{\prime}>\varepsilon_{c}$ for $\alpha=\infty$. The solid line corresponds to the approximation given by Eq. (14).

Summarizing, we have presented numerical and analytical results for the CS states in lattices of coupled identical chaotic maps with interactions that decay with distance as a power law. We have scrutinized the role of the system parameters in the ability of the lattice to attain complete synchronization using various chaotic maps. We observed, in the coupling parameters plane, an overall decrease of the area of the synchronization regions, as the number of coupled maps is increased. Moreover, the shape of these regions is bounded by critical curves which vary with the lattice size in a fashion we were able to predict analytically based on the Lyapunov spectrum of the synchronized state of the lattice, for virtually any chaotic map, with excellent agreement with numerical results. Similar findings have been given for the dependence of the synchronization regions on the degree of chaoticity of uncoupled maps, as well as for the maximal lattice size for which complete synchronization of chaos can be achieved. 


\section{ACKNOWLEDGMENTS:}

We thank Sandro E. de S. Pinto for interesting discussions. This work was partially supported by Brazilian agencies $\mathrm{CNPq}$, FAPERJ, Fundação Araucária and PRONEX.

[1] K. Kaneko, in Theory and Applications of Coupled Map Lattices, edited by K. Kaneko (Wiley, Chichester, 1993).

[2] S. Boccaletti, J. Kurths, G. Osipov, D.L. Valladares and, C.S. Zhou, Phys. Rep. 366, 1 (2002).

[3] K. Kaneko, Physica D 23, 436 (1986).

[4] E. Ott, P. So, E. Barreto and T. Antonsen, Physica D 173, 29 (2002); K. Kaneko, Physica D 41, 137 (1990).

[5] S. Raghavachari and J.A. Glazier, Phys. Rev. Lett. 74, 3297 (1995).

[6] Y. Kuramoto and H. Nakao, Physica D 103, 294 (1997); Y. Kuramoto, D. Battogtokh, and H. Nakao, Phys. Rev. Lett. 81, 3543 (1998).
[7] S. E. de S. Pinto and R. L. Viana, Phys. Rev. E 61, 5154 (2000).

[8] R. L. Viana and A. M. Batista, Chaos, Solitons and Fractals 9, 1931 (1998).

[9] C. Anteneodo, S.E. de S. Pinto, A.M. Batista and R.L. Viana, Phys. Rev. E. 68, 045202(R) (2003) [erratum to include the upper critical line, in press]; see also nlin.CD/0308014.

[10] Y. Kuramoto, Chemical Oscillations, waves and turbulence (Springer-Verlag, Berlin, 1984).

[11] P.M. Gade and C.-K. Hu, Phys. Rev. E 60, 4966 (1999); P.M. Gade, H.A. Cerdeira and R. Ramaswamy, Phys. Rev. E 52, 2478 (1995).

[12] J.-P Eckmann and D. Ruelle, Rev. Mod. Phys. 57, 617 (1985).

[13] G. Rangarajan and M. Ding, Phys. Lett. A 296, 204 (2002).

[14] C. Beck and F. Schlögl, Thermodynamics of chaotic systems (Cambridge University Press, 1993).

[15] R. Toral, C.R. Mirasso, E. Hernández-García and O. Piro, e-print nlin.CD/0002054.

[16] X. Li and G. Chen, IEEE Trans. Circuits Syst. I 50 (2003). 